\title{
Reflectivity of crystalline Ge and Si at the melting temperature measured in real time with subnanosecond temporal resolution
}

\author{
N. Chaoui, ${ }^{\text {a) }}$ J. Siegel, ${ }^{\text {b) }}$ J. Solis, and C. N. Afonso \\ Instituto de Optica, CSIC, Serrano 121, 28006 Madrid, Spain
}

(Received 1 September 2000; accepted for publication 20 December 2000)

\begin{abstract}
Real time reflectivity measurements with subnanosecond time resolution have been used to determine the reflectivity at the melting temperature $R_{S}\left(T_{m}\right)$ of single crystalline $\mathrm{Ge}$ and $\mathrm{Si}$ at 514.5 $\mathrm{nm}$. Due to the excellent time resolution and sensitivity achieved in a single exposure experiment, the reflectivity of the solid just before melting could be measured. Values of $R_{S}\left(T_{m}\right)=0.470$ \pm 0.006 and $R_{S}\left(T_{m}\right)=0.440 \pm 0.008$ for $c$-Ge and $c$-Si have, respectively, been determined. These values, together with those determined by heating in vacuum in the range $300-800 \mathrm{~K}$, are compared to those reported earlier in the literature and the differences are discussed. (c) 2001 American Institute of Physics. [DOI: 10.1063/1.1350413]
\end{abstract}

\section{INTRODUCTION}

The precise knowledge of the evolution of the optical properties of semiconducting materials at high temperatures is very important for many applications, especially those in which laser annealing is one of the key steps of material processing. In spite of the large amount of experimental information available on the temperature dependence of the optical constants of $\mathrm{Ge}^{1,2}$ and $\mathrm{Si}^{3-7}$ in the solid phase, reliable data are in most cases limited to temperatures far below the melting temperature $\left(T_{m}\right)$. This is mostly because the long time high-temperature procedure required for such a determination makes it normally difficult to avoid the transformation of the sample surface (annealing, oxidation) thus hampering the acquisition of reliable data. This problem is even more severe in the case of amorphous materials. ${ }^{8}$ Even for temperatures well below $T_{m}$, the determination of the temperature evolution of optical properties of materials is not an easy task and requires an extremely precise control of the temperature at the surface of the sample. Among others, sophisticated reflectivity/transmitivity techniques ${ }^{3-7}$ or methods such as pseudo-Brewster angle ${ }^{9}$ or polarization modulation ellipsometry ${ }^{5,6}[(\mathrm{PME})$, hereafter $]$ are typically used. In order to minimize surface oxidation effects, the measurements are usually carried out either in ultrahigh vacuum ${ }^{2,3}$ or in an inert gas atmosphere. ${ }^{5,6}$ Despite these precautions, the growth of extremely thin oxide layers may occur and different models have to be used to take into account their effects, particularly for the case of PME. An extensive discussion about the problematic of oxide growth during measurements of temperature dependent optical constants can be found in Ref. 5.

Even when these problems can be successfully solved for temperatures far below the melting temperature, they might become insolvable at temperatures close to (or above) the melting point of a given material. In this context, nano-

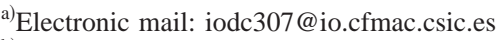

${ }^{b}$ Present address: Femtosecond Optics Group, Physics Department of Imperial College, Prince Consort Road, London, UK.
}

second laser irradiation combined with time resolved measurements have been shown to provide a powerful means to determine the optical constants of molten semiconductors. ${ }^{10,11}$ The main advantage of this procedure is that heating is induced by a short laser pulse over a time lapse sufficiently small to prevent oxidation-related effects giving rise to the formation of a liquid layer that persists long enough (typically in the 10-100 ns range) to allow the use of nanosecond resolution measurements. For instance, the optical constants of liquid $\mathrm{Si}^{10,11}$ and $\mathrm{Ge}^{11}$ at several common laser wavelengths reported by Jellison et al. by means of time resolved ellipsometry, using a $\mathrm{KrF}$ excimer laser for inducing surface melting, are now considered by a majority as reference values.

The complexity of the measurements is even further increased when trying to determine the reflectivity value of the solid phase in the neighborhood of the melting point $\left(T_{m}\right)$. This is because laser induced heating may occur faster than the duration of the laser pulse, since at high energy densities, strong heating can be induced by the leading edge of the pulse, which for the most common high power nanosecond pulsed lasers is just a few nanoseconds. Actually, Jellison et al. ${ }^{12}$ performed real time reflectivity experiments at 633 and $1152 \mathrm{~nm}$ with nanosecond temporal resolution upon excimer laser irradiation $[\lambda=248 \mathrm{~nm}, \tau=40 \mathrm{~ns}$, full width at half maximum (FWHM) $]$ of $c$-Si and $c$-Ge. These measurements allowed them to determine the reflectivity of the hot solid at the melting temperature. They also carried out conventional reflectivity measurements at lower temperatures which showed a linear dependence. However, the values for the reflectivity of the solid at $T_{m}$ measured by real time reflectivity (RTR) were in clear disagreement with the linear extrapolation of the measurements carried at lower temperatures. This discrepancy was explained in terms of solid phase overheating. Nevertheless, Siegel et al. ${ }^{13-15}$ have very recently underlined that a resolution in the nanosecond range can lead to misleading values of the reflectivity whenever the reflectivity changes take place within a few nanoseconds. In this work, we report measurements of the reflectivity of $c$-Ge and $c$-Si in the solid state, at the melting temperature, 
$R_{S}\left(T_{m}\right)$ hereafter, at the $\mathrm{Ar}^{+}$laser line, $\lambda=514.5 \mathrm{~nm}$. These reflectivity values are derived from experiments in which pulsed laser irradiation $(\lambda=584 \mathrm{~nm}, \tau=4 \mathrm{~ns})$ is used to heat the sample surface while the time evolution of its reflectivity is measured in real time with subnanosecond resolution using a streak camera in a single exposure experiment. ${ }^{14} \mathrm{We}$ will show that this high time resolution is necessary even for the relatively long pulse used. Measurements of the temperature dependence of the reflectivity of $c$-Ge and $c$-Si in the $300-800 \mathrm{~K}$ interval using conventional heating techniques have also been carried out for comparison.

\section{EXPERIMENTAL DETAILS}

The samples used were optically polished $\langle 100\rangle, 0.02$ $\Omega \mathrm{cm}, p$-type $\mathrm{Ge}$ wafers and $0.2 \Omega \mathrm{cm}, n$-type $\mathrm{Si}$ wafers. Before irradiation, they were cleaned by successive ultrasonic bathing in different organic solvents (trichloroethylene, acetone, and ethanol) with intermediate and final steps of drying with high purity dry $\mathrm{N}_{2}$. No attempt was made to remove the native oxide layer which was in both cases ( $\mathrm{Ge}$ and $\mathrm{Si}$ ) $2-3 \mathrm{~nm}$ nanometers thick. ${ }^{16}$

The experimental setup used to carry out the RTR measurements with subnanosecond resolution was described in detail in Ref. 14. It essentially consisted of a pump laser used to irradiate the sample surface in a single exposure, and a cw probe laser used to monitor the time evolution of the sample reflectivity. The pump laser was a dye laser amplifier pumped with the second harmonic of a Q-switched $\mathrm{Nd}$ :yttrium-aluminun-garnet laser $(532 \mathrm{~nm})$. The dye amplifier was seeded with the output of a cw dye laser tuned at $584 \mathrm{~nm}$ and pumped with an $\mathrm{Ar}^{+}$laser operating at $514 \mathrm{~nm}$. The amplified output beam $(\lambda=584 \mathrm{~nm}, \tau=4 \mathrm{~ns}$, FWHM) passed through different optical elements for spatial filtering and energy control before being focused at normal incidence onto the sample surface to a spot size of several hundreds of microns. The irradiations were performed in air and each region of the sample was irradiated only once.

The probe beam was delivered by a single mode $\mathrm{cw} \mathrm{Ar}^{+}$ laser $(514.5 \mathrm{~nm})$. Single mode operation of the probe laser turned out to be essential for the measurements with subnanosecond resolution in order to avoid intensity fluctuations in the subnanosecond time scale due to mode competition. ${ }^{14}$ The probe laser beam was pulsed by means of an acoustooptic modulator to a pulse duration of $1 \mu \mathrm{s}$ and then focused to a $\sim 50 \mu \mathrm{m}$ spot size at the center of the irradiated area at an angle of incidence of $15^{\circ}$. The ratio of the pump and probe beam sizes at the sample surface ensured that the region probed by the $\mathrm{Ar}^{+}$laser beam was homogeneously irradiated. The time evolution of the probe beam intensity reflected at the surface was then measured in single exposure by means of a streak camera with a time resolution of $350 \mathrm{ps}$ over a time window with a full width of $50 \mathrm{~ns}$.

The temperature dependence of the reflectivity at 514.5 $\mathrm{nm}$ of the samples was also measured in the temperature interval from 300 to $800 \mathrm{~K}$ using a vacuum chamber (2 $\times 10^{-3} \mathrm{~Pa}$ ) equipped with an ad hoc furnace. The temperature of the sample surface was measured by means of a chromel-alumel thermocouple in contact with it while its
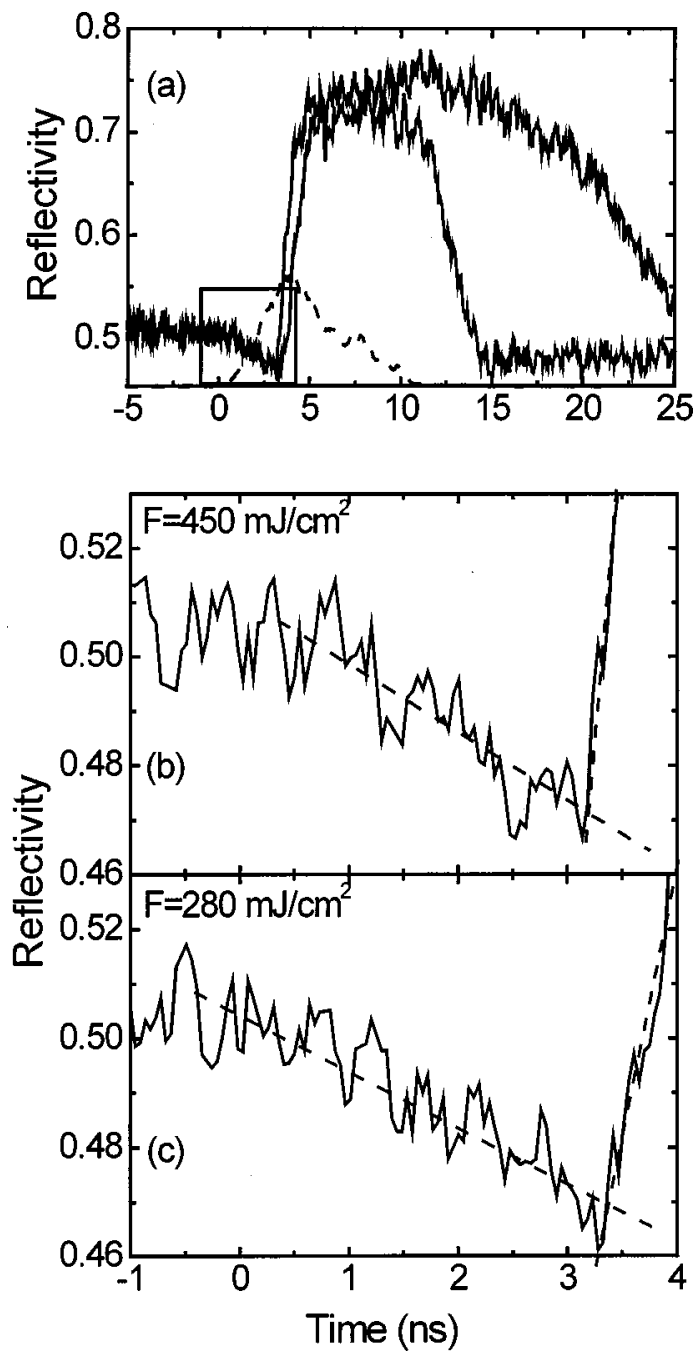

FIG. 1. (a) RTR transients upon nanosecond laser pulse irradiation of $c$-Ge for two laser fluences above the melting threshold together with the profile of the laser pulse (dashed line). (b) and (c) show magnifications of the region of the two transients included in (a) within the square. The dashed lines in (b) and (c) correspond a linear approximation of the evolution of the solid and liquid phase reflectivities at the melt onset.

reflectivity was measured by using the same single mode $\mathrm{cw}$ $\mathrm{Ar}^{+}$laser used in the RTR measurements focused at an angle of $15^{\circ}$ in order to exactly reproduce the conditions of the RTR measurements. The light reflected from the surface was collected by a photodiode connected to a lock-in amplifier. The power of the probe beam was always kept at a low level in order to prevent any possible annealing effects while a small fraction of it was used as a reference to compensate for random, small-amplitude fluctuations of the laser output. The changes of reflectivity, $\Delta R / R(300 \mathrm{~K})=[R(T)-R(300 \mathrm{~K}) /$ $R(300 \mathrm{~K})]$, relative to the value at room temperature $R(300 \mathrm{~K})$ were thus easily determined.

\section{RESULTS}

Figure 1(a) shows the time evolution of the reflectivity of $c$-Ge at $514.5 \mathrm{~nm}$ upon irradiation at two different fluences above the melting threshold $\left[F_{m}(\mathrm{Ge})=200 \mathrm{~mJ} / \mathrm{cm}^{2}\right]$. For both fluences, the absorption of the pump pulse (included as a dashed line), gives rise to a sudden reflectivity 

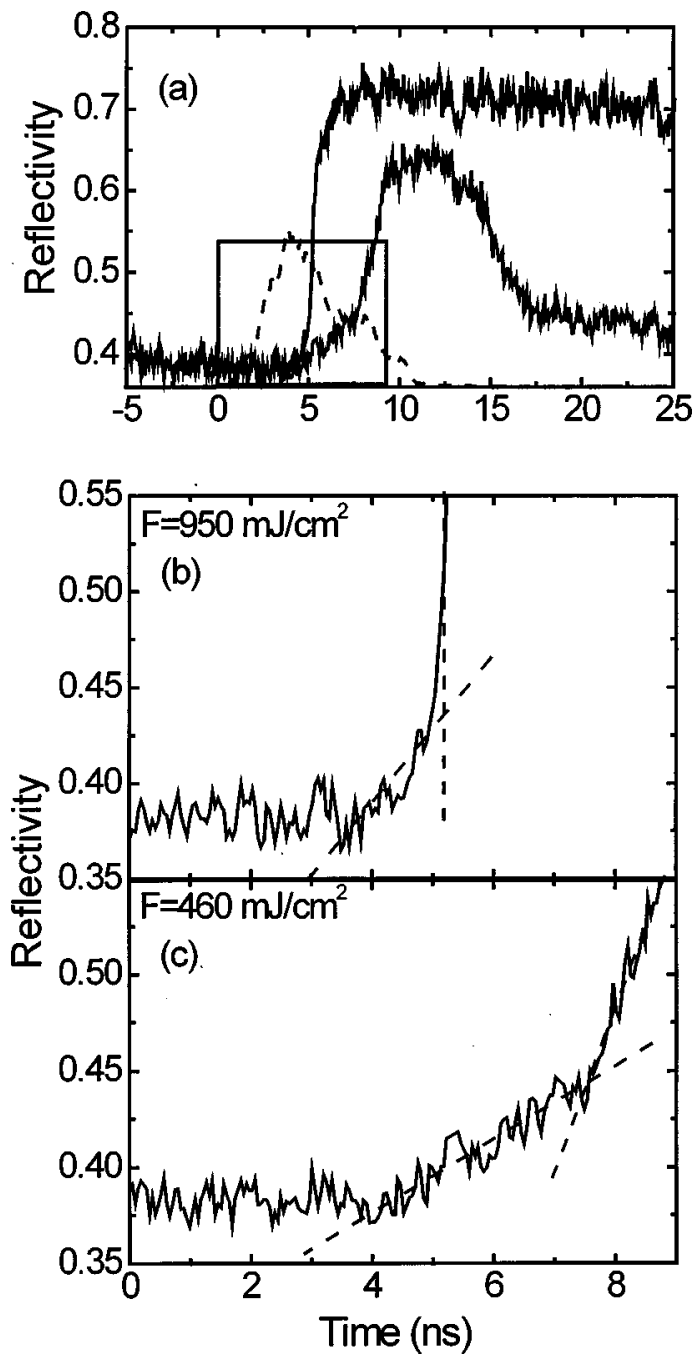

FIG. 2. (a) RTR transients upon nanosecond laser pulse irradiation of $c-\mathrm{Si}$ for two laser fluences above the melting threshold together with the profile of the laser pulse (dashed line). (b) and (c) show magnifications of the region of the two transients included in (a) within the square. The dashed lines in (b) and (c) correspond a linear approximation of the evolution of the solid and liquid phase reflectivities at the melt onset.

increase related to the formation of a metallic liquid layer on top of the surface. ${ }^{12,17}$ If the fluence is sufficient, the reflectivity reaches the value corresponding to an optically thick metallic liquid layer and remains high over several tens of nanoseconds before decreasing again due to the onset of solidification. A similar plot for the case of $c$-Si has been included in Fig. 2(a), although in this case, the melting threshold is higher $\left[F_{m}(\mathrm{Si})=330 \mathrm{~mJ} / \mathrm{cm}^{2}\right]$. In both cases, [Figs. 1(a) and 2(a)], it can be seen that the sharp reflectivity increase associated to the solid-liquid $(\mathrm{S}-\mathrm{L})$ phase transition is much faster than the leading edge of the pump pulse, clearly indicating that even for nanosecond pulse irradiation, a detection technique with subnanosecond resolution can be essential.

Figures 1(b), 1(c), 2(b), and 2(c) show magnifications of the reflectivity increases shown respectively in Figs. 1(a) and 2(a) in the vicinity of the melt onset. For the case of $c$-Ge [Figs. 1(b) and 1(c)], a gentle reflectivity decrease occurs before the onset of the $\mathrm{S}-\mathrm{L}$ phase transition seen as a sharp

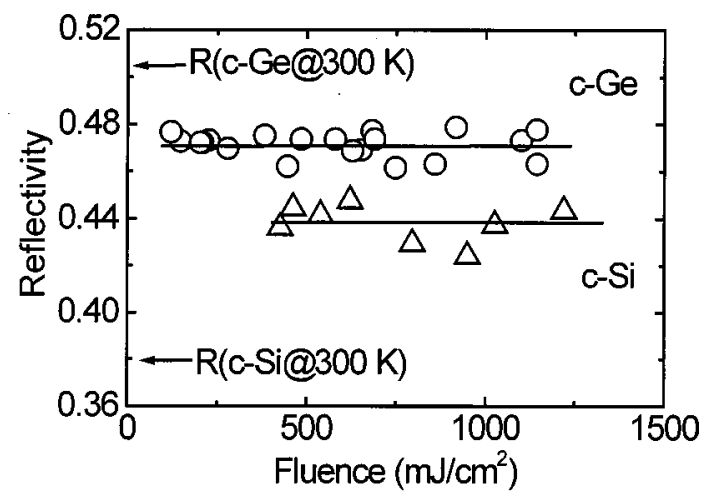

FIG. 3. Reflectivity of the solid phase at the melting temperature as a function of the laser pulse fluence for both $c$-Ge and $c$-Si. The solid lines correspond to the mean value determined and the arrows indicate the reflectivity value at room temperature $(300 \mathrm{~K}): R_{S}(300 \mathrm{~K})=0.505$ for $c$-Ge and $R_{S}(300 \mathrm{~K})=0.380$ for $c-\mathrm{Si}$.

increase. In contrast, the RTR transients of $c$-Si [Figs. 2(b) and 2(c)] exhibit a smooth and continuous increase of the reflectivity before the onset of the $\mathrm{S}-\mathrm{L}$ phase transition seen as a change of slope. We have included as dashed lines the slopes corresponding to the evolution of the reflectivity of the solid and the liquid phases in the neighborhood of the $\mathrm{S}-\mathrm{L}$ transition. The reflectivity values at the interception of the two slopes should correspond to the reflectivity of the solid phase immediately before the phase change and are plotted as a function of laser fluence in Fig. 3. It is clearly seen that within experimental resolution, this reflectivity does not depend on the fluence, confirming that this value corresponds to the reflectivity of the solid at the melting temperature. The mean values respectively obtained for $c-\mathrm{Ge}$ and $c$-Si are $R_{S}\left(T_{m}\right)=0.470 \pm 0.006$ and $R_{S}\left(T_{m}\right)=0.440$ \pm 0.008 , at their melting temperature $\left[T_{m}(c-\mathrm{Ge})=1210 \mathrm{~K}\right.$ and $\left.T_{m}(c-\mathrm{Si})=1683 \mathrm{~K}\right]$. The quoted error values are the mean deviation extracted from a mean square fitting of the data shown in Fig. 3.

Figure 4 shows the temperature dependence of the re-

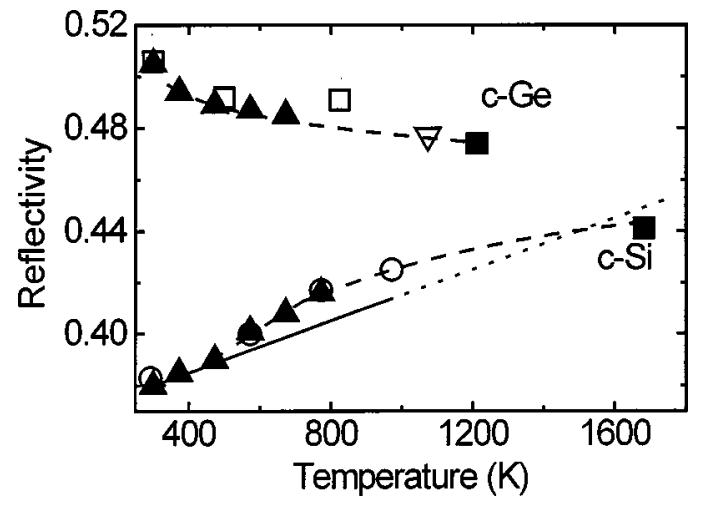

FIG. 4. (\) Reflectivity of $c$-Ge and $c$-Si as a function of temperature in

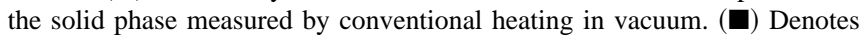
the values of $R_{S}\left(T_{m}\right)$ extracted from the RTR transient at the same wavelength. The long dashed lines correspond to the interpolation of the two sets of results. Data from ( $\square$ ) Viña et al. (Ref. 2), ( $\nabla)$ Aspnes and Studna (Ref. 1), (○) Francois et al. (Ref. 3), and (solid line) Jellison and Modine (Ref. 5) have also been included. The short dashed line is the linear extrapolation of the results in Ref. 5 for temperatures up to $T_{m}$. 
flectivity of $\mathrm{Ge}$ and $\mathrm{Si}$ in the solid phase at the probe wavelength in the temperature interval from 300 to $800 \mathrm{~K}$. The reflectivity values of both materials at the melting temperature $R_{S}\left(T_{m}\right)$ extracted from our RTR measurements have also been included along with the corresponding interpolations (long-dashed lines) from room temperature up to $T_{m}$.

\section{DISCUSSION}

Jellison and co-workers ${ }^{12}$ performed RTR measurements at 632 and $1152 \mathrm{~nm}$ in $c$-Si and $c$-Ge during excimer laser irradiation ( $\lambda=248 \mathrm{~nm}$ and $\tau=40 \mathrm{~ns}$, FWHM). Using nanosecond resolution measurements, they determined the reflectivity of the hot solid before the solid-liquid phase transition at those two wavelengths. For both $c$-Si and $c$-Ge, they found that the reflectivity of the hot solid just before melting laid above the linear extrapolation of the reflectivity of the solid phase measured at lower temperatures. This was interpreted as (i) an overheating of the solid before melting or/ and (ii) a deviation from linearity in the dependence of reflectivity with temperature at high temperatures (above 1000 $\mathrm{K})$. In our case, the independence of the reflectivity of the solid phase immediately before the $\mathrm{S}-\mathrm{L}$ phase transition (Fig. 3) on the laser fluence clearly shows that solid phase overheating, if any, has a negligible effect on the reflectivity of the solid material immediately before the phase transition for pulses as short as $4 \mathrm{~ns}$. Our measurements also show (Figs. 1 and 2) that the $\mathrm{S}-\mathrm{L}$ phase transition upon irradiation with nanosecond laser can be extremely fast (within less than $1 \mathrm{~ns}$ at high fluences), pointing out the need for a sufficient (subnonosecond) time resolution ${ }^{14}$ to determine accurately the reflectivity of the solid phase at $T_{m}$. This observation suggests that, even using a pulse ten times longer than ours, as it was the case in the measurements of Jellison and co-workers, ${ }^{12}$ the time resolution can be a critical issue. This could explain the inconsistency observed by these authors, but also opens up the question of whether the linear extrapolation of the temperature dependence of the solid phase reflectivity is valid for high temperatures, as they assumed in Ref. 12.

The temperature dependence of the reflectivity of semiconductor materials is caused by changes in the band structure due to thermal expansion along with the renormalization of the band energies due to electron-phonon interactions. ${ }^{18}$ The thermal expansion may result in a decrease of the refractive index and thus of the material reflectivity while, at the same time, as the temperature increases, more and more phonons are generated and consequently phonon-assisted indirect transitions are favored, leading to an increase of absorption, which operates in the opposite direction. The balance of both processes, for a given photon energy above the band gap, finally determines the evolution of the reflectivity as a function of temperature. For the particular case of single crystalline $\mathrm{Ge}$ and $\mathrm{Si}$, an extensive discussion about temperature dependence of the optical constants of both materials as function of temperature in a wide photon energy interval can be found in Refs. 2 and 5, respectively. According to the data there provided, in our case, the observed decreasing evolution of $R$ as a function of temperature in Ge is related to the photon energy of the probe beam $(h v=2.41 \mathrm{eV})$ which is close to two important features ${ }^{2}$ of the band structure of $\mathrm{Ge}$, i.e. $E_{1}$ and $E_{1}+\Delta_{1}$ (direct interband transitions in the $\Lambda$ direction of the Brillouin zone). In this region, both real $\left(\epsilon_{1}\right)$ and imaginary $\left(\epsilon_{2}\right)$ parts of the dielectric function exhibit a peak that tends to broaden and to shift to lower energies as the temperature is increased, ${ }^{2}$ explaining the observed decrease of reflectivity with temperature. For the case of $c-\mathrm{Si}$, the PME measurements performed by Jellison and Modine ${ }^{5}$ show that below the $E_{0}^{\prime}$ and $E_{1}$ features, both $\epsilon_{1}$ and $\epsilon_{2}$ monotonically increase with temperature, explaining qualitatively the fact that the reflectivity follows a nearly linear increase for temperatures up to $1000 \mathrm{~K}^{5}$

The comparison of our measurements of the temperature dependence of the reflectivity of $c$-Ge and $c$-Si (up to temperatures below the melting point) with those reported elsewhere ${ }^{1,2,3,5}$ (also included in Fig. 4) shows in general a good agreement, the maximum deviation at a given temperature being in all cases below 2.5\%. For the case of $c$-Ge in the range of temperatures between 300 and $700 \mathrm{~K}$, our measurements show an exponential-like decreasing behavior which is in good agreement with the values reported by Viña et $a .^{2}$ The interpolation of our data, using the reflectivity value we have measured at the melting temperature of $\mathrm{Ge}$ $(1210 \mathrm{~K})$ by means of RTR measurements is, in addition, in excellent agreement with the value reported at $1070 \mathrm{~K}$ by Aspnes and Studna. ${ }^{1}$

For the case of $c-\mathrm{Si}$, the obtained values exhibit a smooth increase whose evolution is in perfect agreement with the results of François et al. ${ }^{3}$ Jellison and Modine ${ }^{5}$ reported that the reflectivity of $c$-Si follows the empirical expression up to $1000 \mathrm{~K}$ for probing wavelengths in the range $410-730 \mathrm{~nm}$

$$
R(\lambda, T)=R(\lambda, T=300 \mathrm{~K})+5 \times 10^{-5}(T-300 \mathrm{~K}) .
$$

The results for $514.5 \mathrm{~nm}$, are also plotted in Fig. 4 where it is seen they are similar to the results obtained in this work for temperatures up to $500 \mathrm{~K}$. For higher temperatures, the slope we experimentally observe is $3 \%$ higher.

Since oxidation effects could be one of the reasons to explain the small differences observed in the case of $c$-Si, it is important to remind that the experiments in Refs. 3 and 5 and the ones here presented were all carried out in wafers in which the native oxide layer was not removed. The measurements in Ref. 3 were performed in ultrahigh vacuum and those in Ref. 5 in an $\mathrm{Ar}-\mathrm{H}_{2}$ atmosphere. Very recently, Heller et al. ${ }^{7}$ have performed very accurate measurements of the reflectivity of $\mathrm{Si}$ at $633 \mathrm{~nm}$ in air for temperatures up to $500 \mathrm{~K}$. The Si surface was intentionally oxidized before the measurements generating $\mathrm{SiO}_{2}$ layers of 2, 20, and $200 \mathrm{~nm}$. These authors showed that an increase of the oxide layer thickness leads to an increase of the slope of the temperature dependence of the reflectivity. Assuming that some oxide formation have occurred during the measurements both in our case and in Ref. 3, the oxide layer thickness required to bring the evolution of the reflectivity quoted in Ref. 5 to a slope similar to that of Ref. 3 above $500 \mathrm{~K}$ and this work would be in the order of $20 \mathrm{~nm}$. It is impossible to produce such a thick layer by annealing at a temperature in the range 
$300-800 \mathrm{~K}$ during less than $1 \mathrm{~h}$ in vacuum $\left(10^{-7} \mathrm{~Pa}\right.$ in Ref. $3,10^{-3} \mathrm{~Pa}$ in our case) and thus surface oxidation cannot account for the differences observed. They could be related to some type of surface modification occurring at temperatures above $500 \mathrm{~K}$ in the slightly reducing atmosphere used in the experiment done by Jellison and Modine. ${ }^{5}$

This comparison also indicates that the discrepancy described in Ref. 12 between the experimental values of $R\left(T_{m}\right)$ (obtained by RTR) and the linear extrapolation of values at lower temperatures was most likely caused by the poor time resolution of the RTR experiment used. Our results show clearly that there are no overheating effects in the solid phase under nanosecond pulse irradiation.

\section{CONCLUSION}

The reflectivity of solid semiconductors $(c-\mathrm{Ge}, c-\mathrm{Si})$ at the melting temperature could be successfully measured with an accuracy better than $2 \%$ using high temporal resolution reflectivity measurements. The main advantage of this single exposure transient technique when compared to conventional ones is that the measurements are performed over a time window that is much shorter than the time required to induce any oxidation effects and thus the experiments can be carried out in air. The comparison of the reflectivity measurements carried out using this heating transient technique to those obtained by conventional heating at lower temperatures indicates that no overheating of the solid occurs and that the dependence of the reflectivity of $c$-Si on temperature departs from the linear dependence proposed earlier by $3 \%$ in the range $500 \mathrm{~K}$ up to $T_{m}$. The measurements of $R_{S}\left(T_{m}\right)$ can be extended to other laser wavelengths as far as the probe laser can provide single mode output. With an improved time resolution, this technique could even provide a measurement of the whole reflectivity evolution from room temperature to the melting point in a single exposure.

\section{ACKNOWLEDGMENTS}

This work has been partially supported by the European Union under Contract No. TMR ERB-CT98-0188. N. Chaoui acknowledges the funding by the same contract. J. Siegel acknowledges the funding of the European Union through the TMR program (ERB-0001GT954352). The authors thank L. Viña from the Universidad Autonoma de Madrid, (Spain), for kindly providing the original data from Ref. 2 .

${ }^{1}$ D. E. Aspnes and A. A. Studna, Rev. Sci. Instrum. 49, 291 (1978).

${ }^{2}$ L. Viña, S. Logothetidis, and M. Cardona, Phys. Rev. B 30, 1979 (1984).

${ }^{3}$ J. C. François, G. Chassaing, L. Argeme, and R. Pierrisnard, J. Opt. 16, 47 (1985).

${ }^{4}$ G. E. Jellison and F. A. Modine, J. Appl. Phys. 53, 3745 (1982).

${ }^{5}$ G. E. Jellison and F. A. Modine, Phys. Rev. B 27, 7466 (1983).

${ }^{6}$ G. E. Jellison and F. A. Modine, Appl. Phys. Lett. 41, 180 (1982).

${ }^{7}$ J. Heller, J. W. Barthe, C. C. Poon, and C. Tam, Appl. Phys. Lett. 75, 43 (1999).

${ }^{8}$ J. Solis and C. N. Afonso, J. Appl. Phys. 72, 2125 (1992).

${ }^{9}$ A. Goldsmith, J. Opt. Soc. Am. 72, 1692 (1982).

${ }^{10}$ G. E. Jellison and D. H. Lowndes, Appl. Phys. Lett. 47, 718 (1985).

${ }^{11}$ G. E. Jellison and D. H. Lowndes, Appl. Phys. Lett. 51, 352 (1987).

${ }^{12}$ G. E. Jellison, D. H. Lowndes, D. N. Mashburn, and R. F. Wood, Phys. Rev. B 34, 2407 (1986)

${ }^{13}$ J. Siegel, J. Solis, and C. N. Afonso, Appl. Phys. Lett. 75, 1071 (1999).

${ }^{14}$ J. Solis, J. Siegel, and C. N. Afonso, Rev. Sci. Instrum. 71, 1595 (2000).

${ }^{15}$ J. Solis, J. Siegel, and C. N. Afonso, Appl. Surf. Sci. 154-155, 449 (2000).

${ }^{16}$ F. Vega, C. N. Afonso, C. Ortega, and J. Siejka, J. Appl. Phys. 74, 963 (1996).

${ }^{17}$ J. Solis and C. N. Afonso, J. Appl. Phys. 69, 2105 (1991).

${ }^{18}$ M. L. Cohen and D. Chadi, in Semiconductors Handbook, edited by M. Balkanski (Amsterdam, North Holland, 1980). 